\title{
树鼠神经肽 $\mathrm{Y}$ 的分子克隆及其灵长类类似物的同源性比较
}

\author{
董 丽 ${ }^{1,2}$, 吕龙宝 ${ }^{1}$, 赖 仞 ${ }^{1, *}$ \\ (1. 中国科学院和云南省动物模型与人类疾病机理重点实验室, 中国科学院昆明动物研究所, 云南 昆明 650223 ; \\ 2. 中国科学院研究生院, 北京 100049)
}

\begin{abstract}
摘要: 树鼠由于与灵长类动物有较密切的亲缘关系和其个体小, 以及繁殖周期短等特性而倍受关注, 尤其是 作为医用实验动物的研究, 近年来已受到越来越多的重视, 但树䳔的分类地位还一直有所争论。该研究从树鼩脑 cDNA 文库中克隆得到编码树鼠神经肽 Y(neuropeptide Y, NPY)前体序列, 序列比对发现该序列与灵长类 NPY 序 列同源性高达 $96.9 \%$ 。将该序列与 GenBank 数据库中其他物种的 NPY 序列构建系统进化树, 发现树鼠与灵长类处 于同一分支。该研究结果揭示了树鼠与灵长类较近的亲缘关系。
\end{abstract}

关键词: 神经肽 $\mathrm{Y}$; 树鼠; 灵长类

中图分类号：Q959.832; Q951.3; Q347 文献标志码：A 文章编号：0254-5853-(2012)01-0075-04

\section{Molecular cloning of Tupaia belangeri chinensis neuropeptide Y and homology comparison with other analogues from primates}

\author{
DONG Li ${ }^{1,2}$, LÜ Long-Bao ${ }^{1}$, LAI Ren ${ }^{1, *}$ \\ (1. Key Laboratory of Animal Models and Human Diseases Mechanisms of the Chinese Academy of Sciences and Yunnan \\ Province, Kunming Institute of Zoology, the Chinese Academy of Sciences, Kunming 650223, China; \\ 2. Graduate University of the Chinese Academy Sciences, Beijing 100049, China)
}

\begin{abstract}
Much attention has been payed to tree shrews for their close phylogenetic relationship with primates, small size, and short reproductive cycle. Especially, they are considered as excellent experiential animals for medicine or/and disease research. A nucleotide sequence encoding neuropeptide Y(NPY) precursor has been cloned from the cDNA library of Tupaia belangeri chinensis. Sequence alignment revealed that the sequence homology with primate NPY was up to $96.9 \%$. The phylogenetic analysis based on NPY precursor sequence revealed that the tree shrew has a close relationship with primates.
\end{abstract}

Key words: Neuropeptide Y; Tupaia belangeri chinensis; Primates

神经肽 Y(neuropeptide Y, NPY) 是 Tatemoto et al (1982)首次从猪脑中提取纯化出的一种具有生物 活性的内源性信息物质，由 36 个氨基酸组成，相对 分子质量为 $4.2 \times 10^{3}$ 。在结构和功能上与胰多肽 (avian pancreatic polypeptide, APP) 和肽 YY(peptide YY, PYY)非常相似，所以将其划分为胰多肽家族 (Tatemoto et al,1982)。NPY 序列 N 端和 C 端各有一 个酪氨酸残基, $\mathrm{C}$ 端酪氨酸残基存在酰胺化修饰
(Allen et al,1987), 这与生物体内许多活性肽(如促 性腺激素 GnRH)相同(Cerdá-Reverter \& Larhammar, 2000)。这一修饰不仅可保护 $\mathrm{C}$ 端免受羧肽酶降解, 同时与生物活性密切相关。 $\mathrm{N}$ 端的酪氨酸残基对于 NPY 三级结构的稳定及与 NPY 受体的结合非常重 要(Tatemoto et al,1982)。

在细胞内, NPY 以前体方式合成, 前体由 97 98 个氨基酸组成, $\mathrm{N}$ 端 $28 \sim 29$ 个氨基酸为信号肽,

收稿日期: 2011-10-31；接受日期：2011-12-29

基金项目: 中国科学院基础前沿研究专项项目(KSCX2-EW-R-11, KSCX2-EW-J-23); 中国科学院和云南省动物模型与人类疾病机理重点实验室资 助项目

*通信作者(Corresponding author), E-mail: rlai@mail.kiz.ac.cn 
前体翻译后经过酶切、 $\mathrm{C}$ 端酪氨酸酰基化修饰后成 为具有生物活性的 36 个氨基酸组成的活性肽 (Higuchi et al,1988)。NPY 在进化过程中高度保守， 哺乳动物中, 人以及大鼠、豚鼠的成熟 NPY 氨基酸 序列完全相同，而猪的 NPY 也只是在第 17 位氨基 酸有所不同, 鱼类电鳐目黑斑电鳐(Torpedo marmorata) 有 33 个氨基酸与哺乳动物 NPY 序列中 一致, 只有另外 3 个不同(Pedrazzini et al, 2003)。

NPY 最初从哺乳动物中分离得到, 之后的研究 发现, NPY 不仅在哺乳动物中表达, 也广泛分布于 鸟类、鱼类和两栖类。在体内, 神经肽作为一种神 经递质参与体内多种生理调节过程。NPY 可直接对 下丘脑活动产生作用, 影响多种因子与激素的合成 和分泌(Danger et al,1990; Breton et al,1991); NPY 可 促进机体的摄食行为, 调节机体能量平衡(Kuo et al, 2007); 调节血管收缩, 具有增强血管紧张素 II(AngII)、内皮素和交感缩血管物质(NE)等血管收 缩物质而抑制舒血管物质的作用, 并且与免疫功能 也有着密切关系(Zukowska et al, 2003)。除成熟肽 以外, 前体加工过程中 $\mathrm{C}$ 端切割产生的 30 个氨 基酸组成的肽段(CPON)也非常保守。有研究表 明, CPON 广泛分布于中枢和外周神经系统中, 并且与 NPY 在神经细胞中有相同的定位 (Gulbenkian et al,1985; Marti et al,1990)。这似乎 表明, CPON 并不仅仅是 NPY 加工过程中的一 个“副产物”, 它可能还发挥着其他的生物学功 能, 遗憾的是, 目前仍没有发现该肽段的生物 学功能。

树鼠是一种小型哺乳动物, 由于与灵长类相近, 近年来受到越来越多科学工作者的关注。该文采用 树鼠脑组织为材料, 构建 cDNA 文库, 以 PCR 方法 扩增得到一条编码树鼣 NPY 前体的 cDNA 序列。

\section{1 材料与方法}

\section{1 树鼠脑 cDNA 文库的构建}

取成年树獡(Tupaia belangeri, 来自昆明动物 研究所实验动物中心) 1 只, 麻醉剂麻醉后, 解剖头 部取出整个脑组织, 立即放入液氮中保存备用。总 RNA 提取采用 Trizol 试剂(invitrogen), 参照试剂说 明书进行。cDNA 双链合成采用 CLONTECH 公司 SMART $^{\mathrm{TM}}$ cDNA Library Construction Kit 试剂盒。 一链使用 CDSIII 引物 5'-ATTCTAGAGGCCG $A G G C G G C C G A C A T G-\mathrm{d}(T)_{30} \mathrm{~N}_{-1} \mathrm{~N}-3^{\prime}(\mathrm{N}=A 、 G 、 C 、$
$T ; \mathrm{N}_{-1}=A 、 G 、 C$ )和 $\mathrm{SmartV}$ 寡聚核苷酸 5'- $A A G C A$ GTGGTATCAACGCAGAGTACGCGGG-3'合成; 二 链使用 5'PCR 引物 5'-AAGCAGTGGTATCAACG $C A G A G T-3$ '合成。

\section{2 克隆篮选}

对上一步构建的 cDNA 文库进行随机克隆篮 选, 根据构建 cDNA 文库时 5'端和 3'端通用序列设 计引物, 上游引物和下游引物分别为: 5'-AAGCAGTGGTATC-AACGCAGAGT-3'和5'ATTCTAGAGGCCGAGGCGGCCGACATG-3'，引物 由上海生物工程有限公司合成。以上一步 smart 合 成双链 $\mathrm{cDNA}$ 为模板进行 $\mathrm{PCR}$ 。反应条件如下: 首 先 $95{ }^{\circ} \mathrm{C}$ 变性 $5 \mathrm{~min}$; 接下来 $95^{\circ} \mathrm{C}$ 变性 $30 \mathrm{~s}, 55^{\circ} \mathrm{C}$ 退火 $30 \mathrm{~s}, 72{ }^{\circ} \mathrm{C}$ 延伸 $1 \mathrm{~min}, 30$ 个循环; 最后 $72^{\circ} \mathrm{C}$ 延伸 $10 \mathrm{~min}$ 。PCR 产物琼脂糖凝胶电泳检测, 切胶 回收, 克隆到 PMD19-T 载体(TaKaRa 公司), 选择 103 个阳性克隆测序。

\section{3 克隆序列与其他物种神经肽 $Y$ 前体序列比对}

克隆得到的序列, 采用美国国家生物技术信息 中心 (NCBI, National Center for Biotechnology Information, http://www.ncbi.nlm.nih.gov) 网站的 BLAST 软件, 将测序后获得的 cDNA 序列与 GenBank 数据库中已公布的序列进行同源性比对。

\section{4 树鼠神经肽 Y 前体序列分子进化分析}

根据 BLAST 比对结果, 从 GenBank 中下载其 他物种神经肽 Y 前体序列, 采用 MEGA5.0 软件 (Tamura et al, 2011) 邻近归并法(neighbor joining method)构建系统进化树。

\section{2 结 果}

\section{1 树鼠脑 cDNA 文库构建}

提取的 RNA 经紫外分光光度计及 $1 \%$ 琼脂糖凝 胶电泳检测, 结果显示 RNA 浓度为 $882 \mathrm{ng} / \mu \mathrm{L}$, $\mathrm{OD}_{260} / \mathrm{OD}_{280}$ 值为 2.01 , RIN 值为 $8.28 \mathrm{~S} / 18 \mathrm{~S}$ 值 1.7 , 各指标均符合后续建库要求。以所提 RNA 按照 SMART $^{\mathrm{TM}}$ cDNA Library Construction Kit 试剂盒操 作手册建立 cDNA 文库，电泳检测显示 250 2 000 $\mathrm{bp}$ 位置有连续弥散状, 符合说明书要求, 可作为模 板进行后续实验。

\section{2 树鼠 NPY 序列克隆及序列比对}

将 PCR 扩增所得序列测序, 得到一条长度为 291 bp 的核苷酸序列，其核苷酸序列如图 1 所示。 经 Blast 比对, 此片段与数据库中人类 NPY 前体序 
列非常类似，推测该序列编码蛋白为树鼣中 NPY atgctaagtagcaagtgtctgggactatccggactgaccetcgccetgtccetgctcgtg \begin{tabular}{llllllllllllllllllll}
$M$ & $L$ & $S$ & $S$ & $K$ & $C$ & $L$ & $G$ & $L$ & $S$ & $G$ & $L$ & $T$ & $L$ & $A$ & $L$ & $S$ & $L$ & $L$ & $V$ \\
\hline
\end{tabular} tgcttgggtgctctgggcgaggcataccctccaagccegacaacccgggcgaggacgcg \begin{tabular}{llllllllllllllllllllll} 
C & L & G & A & L & $G$ & E & A & Y & P & S & K & P & D & N & P & G & E & D & A \\
\hline
\end{tabular} ccggcggaggacatggccagatactactcggcgctgcgacattacatcaacctcatcacc \begin{tabular}{|llllllllllllllllllll|}
\hline $\mathrm{P}$ & $\mathrm{A}$ & $\mathrm{E}$ & $\mathrm{D}$ & $\mathrm{M}$ & $\mathrm{A}$ & $\mathrm{R}$ & $\mathrm{Y}$ & $\mathrm{Y}$ & $\mathrm{S}$ & $\mathrm{A}$ & $\mathrm{L}$ & $\mathrm{R}$ & $\mathrm{H}$ & $\mathrm{Y}$ & $\mathrm{I}$ & $\mathrm{N}$ & $\mathrm{L}$ & $\mathrm{I}$ & $\mathrm{T}$ \\
\hline
\end{tabular} aggcagagatatggaaaacgatctagtcccgagaccttgatttcagatctcttgatgaga

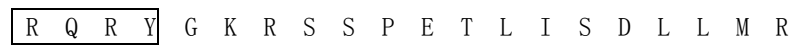
gaaagcacagaaaatgttcccagaacaaggcttgaagacccttctatgtgg

$\begin{array}{llllllllllllllllll}\text { E } & \mathrm{S} & \mathrm{T} & \mathrm{E} & \mathrm{N} & \mathrm{V} & \mathrm{P} & \mathrm{R} & \mathrm{T} & \mathrm{R} & \mathrm{L} & \mathrm{E} & \mathrm{D} & \mathrm{P} & \mathrm{S} & \mathrm{M} & \mathrm{W}\end{array}$

图 1 树鼠脑组织 cDNA 文库中克隆所得 NPY 核苷酸 序列及对应氨基酸序列

Fig.1 The nucleotide sequence encoding neuropeptide Y (NPY) and corresponding amino acid sequence from Tupaia belangeri

下划线表示信号肽序列; 方框表示成熟肽序列。

The underlined amino acid sequence is signal peptide; the boxed amino acid sequence is mature peptide.
前体。291 bp 为树舄 NPY 前体序列编码区, 可编码 97 个氨基酸组成的 NPY 前体。其中前 84 bp 核苷 酸序列编码 28 个氨基酸组成的信号肽, 中间 $108 \mathrm{bp}$ 核甘苷酸编码长度为 36 个氨基酸的成熟肽。

\section{3 树鼠 NPY 前体序列与其灵长类类似物同源性 比较}

将克隆得到树鼣 NPY 前体序列与 GenBank 数 据库灵长类中狝猴 (Macaca mulatta 登录号 NP_001027986.1)、人 (Homo sapiens 登录号 AAA59944.1)NPY 前体序列进行同源性比对。结果 显示, 树鼣 NPY 前体序列成熟肽 36 个氨基酸与灵 长类 3 个物种完全一致, 信号肽部分 28 个氨基酸也 仅在第 3、6、26 位存在 3 个氨基酸的差异, 整个前 体肽序列 97 个氨基酸中有 94 个氨基酸完全一致, 同源性高达 96.9\%。

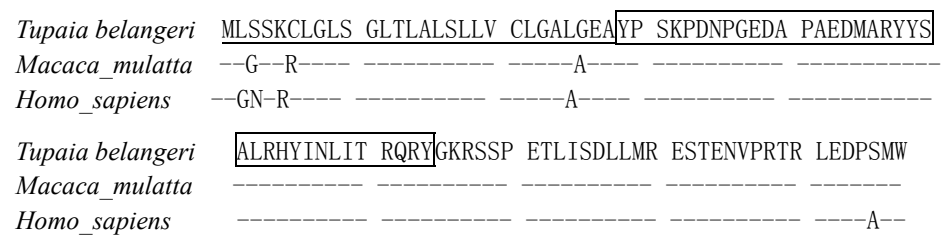

图 2 树鼠 NPY 前体序列与其灵长类类似物序列比对

Fig.2 Comparison of Tupaia belangeri NPY precursor with Primates NPY precursors 下划线表示信号肽序列; 方框表示成熟肽序列; “-”表示相同氨基酸。

The underlined amino acid sequence is signal peptide; the boxed amino acid sequence is mature peptide; Gaps (-) indicate the same amino acid residue.

\section{4 树鼠神经肽 $Y$ 前体序列分子进化分析}

将克隆所得树鼠 NPY 前体与人(Homo sapiens, GenBank 登录号 AAA59944.1)、猕猴 (Macaca mulatta, NP_001027986.1)、褐 家 鼠 (Rattus norvegicus, NP_036746.1)、小家鼠(Mus musculus, NP_075945.1)、红原鸡(Gallus gallus, NP_990804.1)、 家鸽 (Columba livia, ACB86930.1)、非洲爪蟾
(Xenopus laevis, AAA49914.1)、热带爪蟾(Xenopus tropicalis, AAI35388.1)、七鳃鳗(Lampetra fluviatilis, AAA21352.1)、金鱼(Carassius auratus, P28672.1)、 锦鲤(Cyprinus carpio, Q9DGK7.1)、黄牛(Bos taurus, AAR37328.1)、白项长臂猿(Nomascus leucogenys, XP_003270460.1)、绵羊(Ovis aries, CAD10677.1) NPY 前体序列构建系统进化树, 从图 3 可以看出,

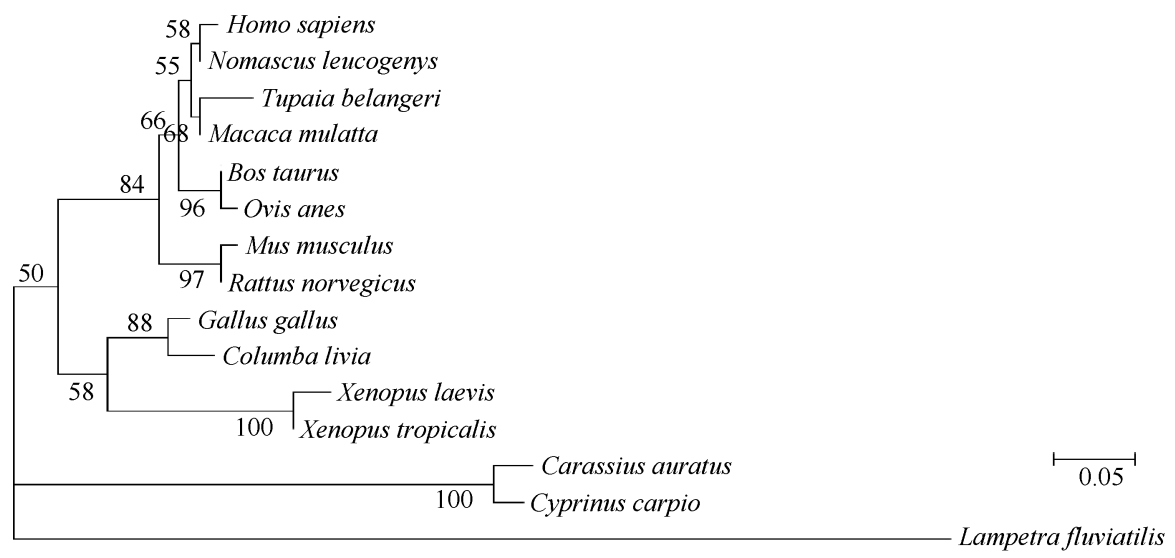

图 3 神经肽 Y(NPY)氨基酸序列系统进化树

Fig.3 Evolutionary tree of Neuropeptide $\mathrm{Y}$ amino acid sequence 
NPY 虽然在进化过程中序列高度保守, 但也存在种 属特异性。树舄与灵长类处于同一分支, 说明两者 亲缘关系较近。

\section{3 讨 论}

NPY 在生物体中分布广泛，目前通过分离纯 化和克隆手段已经在多个物种中发现其类似物的 存在, 包括冷椎动物和无脊椎动物(Blomqvist et al, 1992)。通过不同物种间的序列比对发现, NPY 序列 高度保守, 特别是发挥生物学活性的成熟肽序列部 分。本研究从树鼠脑组织 cDNA 文库中克隆得到了 一条相似的序列, 推测该序列在树鼠体内发挥着和 其他物种类似的生物学功能。

长久以来，树鼠的系统分类地位一直存在争议， 有人主张将其归入食虫目, 有人认为应该属于灵长 目, 后来又有人提出它们既不属于灵长目, 也不属

\section{参考文献:}

Allen J, Novotný J, Martin J, Heinrich G. 1987. Molecular structure of mammalian neuropeptide $\mathrm{Y}$ : analysis by molecular cloning and computer-aided comparison with crystal structure of avian homologue[J]. Proc Natl Acad Sci USA, 84(8): 2532-2536.

Blomqvist AG, Söderberg C, Lundell I, Milner RJ, Larhammar D. 1992. Strong evolutionary conservation of neuropeptide $\mathrm{Y}$ : sequences of chicken, goldfish, and Torpedo marmorata DNA clones[J]. Proc Natl Acad Sci USA, 89(6): 2350-2354.

Breton B, Mikolajczyk T, Popek W, Bieniarz K, Epler P. 1991. Neuropeptide $\mathrm{Y}$ stimulates in vivo gonadotropin secretion in teleost fish[J]. Gen Comp Endocrinol, 84(2): 277-283.

Cerdá-Reverter JM, Larhammar D. 2000. cNeuropeptide Y family of peptides: structure, anatomical expression, function, and molecular evolution[J]. Biochem Cell Biol, 78(3): 371-392.

Chen SY, Xu L, Lü LB, Yao YG. 2011. Genetic diversity and matrilineal structure in Chinese tree shrews inhabiting Kunming, China[J]. Zool Res, 32(1): 17-23. [陈仕毅, 许凌, 吕龙宝, 姚永刚. 2011. 昆明城郊 中国树鼠群体线粒体 DNA 遗传多样性[J]. 动物学研究, 32(1): 17-23.]

Danger JM, Tonon MC, Jenks BG, Saint-Pierre S, Martel JC, Fasolo A, Breton B, Quirion R, Pelletier G, Vaudry H. 1990. Neuropeptide Y: localization in the central nervous system and neuroendocrine functions[J]. Fundam Clin Pharmacol, 4(3): 307-340.

Gulbenkian S, Wharton J, Hacker GW, Varndell IM, Bloom SR, Polak JM. 1985. Co-localization of neuropeptide tyrosine (NPY) and its C-terminal flanking peptide (C-PON)[J]. Peptides, 6(6): 1237-1243.

Higuchi H, Yang HY, Sabol SL. 1988. Rat neuropeptide Y precursor gene
于食虫目，而是一个独立的目，称为攀鼠目(Chen et al, 2011; Peng et al, 1991)。本研究通过克隆所得的 树舄 NPY 前体序列与灵长类类似物进行同源性比 对发现，树鼠 NPY 成熟肽序列与其他灵长类完全 吻合, 信号肽序列也仅存在 3 个氨基酸的差异. 但 同时将灵长类 NPY 前体序列与其他物种进行比对 发现, NPY 成熟肽高度保守, 所以单从成熟肽序列 不能判断树舄与灵长类之间亲缘关系。不过虽然各 物种间 NPY 成熟肽序列高度保守，但信号肽部分 保守程度却较低, 存在种属特异性, 因此, 本研究 选用 NPY 前体序列构建系统进化树来进行研究。 从构建的进化树(图 3)可以看出, 该树准确定位了 鱼类、两栖类、鸟类和哺乳类的亲缘关系，说明以 NPY 前体序列构建的系统进化树具有较高的可信 度。从该进化树还可以看出, 树鼠与灵长类亲缘关 系更近, 说明树鼠与灵长类更相似。 expression. mRNA structure, tissue distribution, and regulation by glucocorticoids, cyclic AMP, and phorbol ester[J]. J Biol Chem, 263(13): 6288-6295.

Kuo L E, Kitlinska JB, Tilan JU, Li L, Baker, SB, Johnson MD, Lee EW, Burnett MS, Fricke ST, Kvetnansky R, Herzog H, Zukowska Z. 2007. Neuropeptide $\mathrm{Y}$ acts directly in the periphery on fat tissue and mediates stress-induced obesity and metabolic syndrome[J]. Nat Med, 13(7): 803-811.

Marti E, Bello AR, Lancha A, Batista MA. 1990. Neuropeptide tyrosine (NPY) and its C-terminal flanking peptide (C-PON) in the developing and adult spinal cord of a reptile[J]. J Anat, 172: 149-156.

Pedrazzini T, Pralong F, Grouzmann E. 2003. Neuropeptide Y: the universal soldier[J]. Cell Mol Life Sci, 60(2): 350-377.

Peng YZ, Ye ZZ, Zou RJ, Wang YX, Tian BP, Ma YY, Shi LM. 1991. Biology of Chinese Tree Shrew[M]. Kunming, China: Yunnan Science and Technology Press. [彭燕章, 叶智彰, 邹如金, 王应祥, 田保平, 马原野, 施立明. 1991. 树獡生物学. 昆明: 云南科技出版社.]

Tatemoto K, Carlquist M, Mutt V. 1982. Neuropeptide Y--a novel brain peptide with structural similarities to peptide $\mathrm{YY}$ and pancreatic polypeptide[J]. Nature, 296(5858): 659-660.

Tamura K, Peterson D, Peterson N, Stecher G, Nei M, Kumar S. 2011. MEGA5: molecular evolutionary genetics analysis using maximum likelihood, evolutionary distance, and maximum parsimony methods[J]. Mol Biol Evol, 28(10): 2731-2739.

Zukowska Z, Pons J, Lee EW, Li LJ. 2003. Neuropeptide Y: a new mediator linking sympathetic nerves, blood vessels and immune system?[J]. Can J Physiol Pharmacol, 81(2): 89-94. 